\title{
Novel FUS gene variants linked to essential tremor
}

Research conducted in Chinese and Canadian populations has added to the growing evidence that mutations in the fused in sarcoma (FUS) gene can constitute genetic risk factors for essential tremor (ET), a common adultonset neurological disorder characterized by postural and/ or kinetic tremor. The new work builds on the recent discovery of a FUS nonsense mutationGln290Stop-that segregated with ET in a large Canadian family.

In a study involving a total of 7,548 individuals of Chinese ethnicity, Eng-King Tan and colleagues found a novel FUS gene variant, Met392Ile, that was associated with a significantly elevated risk of ET. In a control cohort of patients with Parkinson disease, the frequency of this mutation was no higher than in the general population.

"Interestingly, the new polymorphic risk variant we identified is totally absent in the white population-both ET and healthy controls-whereas the pathogenic mutation reported in the original ET family was absent in our samples," says Tan. “This finding suggests the existence of possible ethnicity-specific FUS mutations in ET."

In a separate study, Carles Vilariño-Güell and colleagues sequenced the FUS gene in 217 Canadian patients with ET. The researchers identified a heterozygous Arg377Trp substitution in one patient who also had a family history of ET. No DNA samples were available from the other affected family members, but in silico analysis indicated that the variant is potentially deleterious.

"Functional work in cellular and animal models is paramount to identify the biological outcome of the Arg377Trp mutation," explains Vilariño-Güell. "Confirmation of pathogenicity and the development of physiologically relevant models of ET will provide a better understanding of the molecular mechanisms implicated in the onset of disease."

The FUS gene is of considerable interest to neurologists, given its previously described links with conditions such as frontotemporal dementia and amyotrophic lateral sclerosis. The findings of Tan, Vilariño-Güell and co-workers, if validated, could open up new avenues of investigation into the role of this gene in ET and other neurological diseases.

Heather Wood

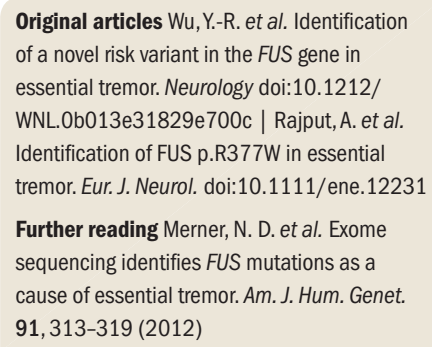

